\title{
Media Skills Training Intervention Based on Automated Recognition of Human Emotion and Non-Verbal Behaviour
}

\author{
Monica Pereira \\ Brunel University London \\ Kingston Lane, London, Uxbridge UB8 3PH \\ monica.pereira@brunel.ac.uk
}

\begin{abstract}
Across multiple sectors training programmes aim to help learners improve their communication skills. It is well recognised that non-verbal 'social signals' play an important role in communication effectiveness. Previous research in the social signalling domain meticulously observed hours of videos and conducted observational studies to identify these social signals. This resulted in subjective inferences about human emotions. The aim of the current research is to investigate whether social signals can be detected and trained using automated technology in a person-toperson training context in three stages; exploratory stage, feedback design stage and an experimental stage. This research will allow trainers to provide objective feedback to trainees about their performance with a clear criterion. This research will also explore the best way to feedback signals detected and whether this is effective and actionable. Further long-term benefits of this research might contribute to the eventual development of a training avatar.
\end{abstract}

Social Signals. Training. Automated Affect Technology. Non-verbal Behaviour.

\section{CONTEXT AND MOTIVATION}

Effective communication is the key to conveying a clear and concise message in a media interview, particularly in hostile or crisis situations (Taylor, 2015). Communication is a complex phenomenon but it simply defined as the transmission of information from one person to another (Fiske, 2010). More specifically, social signals are the emotionally informative signals that directly or indirectly provide meaning around social interactions (Vinciarelli, Pantic and Bourlard, 2009; Poggi and D 'errico, 2011; Tracy et al., 2015). There is emerging evidence for the importance of non-verbal cues / social signals in conveying effective messages (Tracy et al., 2015).

Earlier research in the social signalling / non-verbal cues domain relied solely on meticulous observation and analysis of video data, such as viewing hours of recorded videos in order to interpret social situations (Vrij, Edward and Roberts, 2000).This method of behaviour analysis does not scale with large amounts of data. By utilising the recently developed automated affect technology in the current research, a scalable alternative that gives rise to the possibility of faster and more objective measurement of social signals is proposed.
This research investigates whether off-the-shelf technology can detect the social signals (nonverbal cues) that are essential for a media interview and whether these signals can be trained using this technology. It also investigates the most appropriate method for providing effective feedback to trainees about their performance.

\section{BACKGROUND}

Social signals are defined as a perceivable stimulus produced by the sender and perceived by the receiver. Humans produce these signals in all modalities such as intonations, gestures, prosody (McNeill, 1992; Kendon, 2004), posture (Condon and Ogston, 1966), head movements (Cerrato, 2005), facial expression (Ekman and Friesen, 1972), gaze (Marzillier, 1976), physical contact and spatial behaviour (Hall, 1969).

Emotions are thought to be embedded in communication channels and are said to be unconsciously transmitted via social signals / nonverbal signals area which is often referred to as a second string of communication or honest signals (Pentland and Heibeck, 2008; Hurley and Frank, 2011). There are four honest signals that are argued to be consistently present in all social interactions: mimicry (mirroring), influence, activity and 
consistency (Pentland and Heibeck, 2008). Additionally, there is six basic emotions that are observed cross-culturally which are joy, sad, fear, anger, disgust and surprise (Ekman \& Friesen, 1982). The facial expressions which can be understood universally are the 6 basic emotions in addition to contempt (Ekman, 1992). There is some debate whether surprise is an emotion as it is a cognitive component that may be present with any emotion (Oatley and Johnson-Laird, 1987), i.e. winning an award for best performance when the performance poor, the emotions felt would be joy and surprise.

The accuracy of humans in detecting social signals are uncertain (Bond $\mathrm{Jr}$ and DePaulo, 2006). This is because detection of social signals is subjective (Bush et al., 1989). This results in subjective feedback given to trainees in a training context. Research has shown that the capability of experts in the field of non-verbal communication is also underestimated (Frank and Feeley, 2003). Further evidence for this is that individuals could not detect the difference between facial expressions of pain or no pain even after training (Bartlett et al., 2014). Together, these studies illustrate that training in detecting social signals are complex due to its subjective nature.

The use of automated technology in detecting nonverbal behaviour in the context of interview performance has been explored. A study used of automated technology to explore the non-verbal behaviour that is present in a good university entry interview (Naim et al., 2015). Performance was judged by observers later. It was reported that smiling more is perceived as good (Naim et al., 2015). This study illustrates that the use of automated technology can be used to detect nonverbal behaviour.

The use of automated technology could enable trainers to use software to provide visual feedback to participants to highlight areas of improvement. The method in which feedback is presented is important as it could have a negative impact on trainees' performance. There are a number of visual elements in graphical displays that excise cognitive resources which result in an increase in cognitive load, such as divided attention (Bennett and Flach, 1992).

Visual displays which allow the observer to utilise efficient processes of perception and pattern recognition instead of extensive use of cognitive processes of memory, integration and inference are more effective in learning and information retention (Rasmussen and Vicente, 1989; Vicente and Rasmussen, 2014). By mapping multiple process variables into a single geometric form, such as an octagon, this enables high-level visual properties such as symmetry which reduces divided attention (Woods, 1991).

The extent to which humans can be trained using automated technology has been explored very little. A study by Damian et al. (2015) investigated the effectiveness of virtual interview training for children for a job interview in a gaming context and compared it to conventional training. The training avatar proved more effective in this study than the conventional training.

Research illustrates that automated technology may be used in detecting the non-verbal cues / social signals in the context of a media interview and it may be possible to be used in a training context.

\section{RESEARCH OBJECTIVE AND AIM}

The aim of this research is to explore whether communication skills can be enhanced using off-theshelf emotion recognition technology detect social signals in the context of media skills training. In addition to this, the most appropriate method of presenting feedback to participants using automated technology is also explored. This research is necessary to investigate as human-human training tends to be subjective (Vrij et al, 2000).

The current research aims to objectively detect the social signals that are currently proposed as necessary for a media interview as these are essential for effective communication. It eliminates several concerns associated with human-human training by providing objective feedback to trainees, ensuring expected training outlines are met, defining a clear criterion. This would also make training easily available to learners and potentially promotes deeper learning. As a result, training authenticity may improve. The overall research question can be defined as:

Can communication skills be improved using automated technology in media skills training?

The aim of this paper is to outline the overall approach taken to address this research question.

\section{RESEARCH APPROACH}

This research was conducted in three stages to assess the proposed research question; an exploratory (explored the social signals associated with a good media interview), a design (identified the best method of social signal feedback) and an experimental stage (compared whether social signal feedback is more effective than standard feedback). The communication channels assessed in this research were facial expression, voice emotions and honest signals. The technology used 
to detect communication channels were utilised in the exploratory and experimental stages.

\subsection{Automated Detection of Communication Channels}

The software that was used to detect facial expression was AFFDEX by Affectiva. This software uses a Facial Analysis Coding System (FACS) that produces the seven facial expressions that are observed universally (Ekman and Friesen, 1978). Additional expressions were assessed as they were important for a media interview. These were brow furrow, smiling and smirking (Taylor, 2015).

The Shimmer 3 was used to detect gestures and physiological recordings such as heart rate. Both the facial recognition software and the Shimmer 3 were recorded on iMotions biometric research platform.

The software that was used to analyse voice emotion was Layered Voice Analysis / Nemesysco ${ }^{\text {ttd }}$. This software detects the genuine emotions that are sensitive to the speaker (Nemesysco.com). The emotions that are produced are energy, content, upset, angry, stress, embarrassment, intensive thinking, imagination activity, hesitation, uncertainty, excitement, concentration, arousal, extreme state, overall cognitive activity and rationality.

Sociometric badges were used to record a social interaction. They have been designed by Pentland and Heibeck (2010) to analyse face-face social interactions. They have been designed to detect honest signals: mimicry (mirroring), influence, activity and consistency.

\subsection{Subjective Rating Scale and Usability Scale}

The Conversation Skills Rating Scale (CSRS) was used as a measure of communication performance for each interview (Spitzberg and Adams, 2007) for the exploratory and experimental stages. The CSRS contains two rating sections; a 25 -item scale rating verbal and non-verbal communication and a 5-item scale which measures communication skills. The communication skill items were used. The communication skills items have a high internal consistency above $\alpha=.85$ in an interview setting. In the instance of multiple raters, a typical inter-rater reliability is $\alpha=.75$ (Ellis, Duran and Kelly, 1994).

The System Usability Scale (SUS) was used to gather participants' views on ease of use for the different methods of presenting feedback to participants about their performance in the design stage. The SUS is a reliable 10-item questionnaire with 5 responses for each ranging from 'Strongly Agree' to 'Strongly Disagree' (Brooke, 1996). It has been accurate in identifying the usability of a range of different systems, such as websites and software (Kortum and Bangor, 2013).

\subsection{Exploratory Stage}

The aim of the exploratory stage is to explore whether automated technology can detect which social signals are most promising for evaluating participant performance. This was done by observing a typical media skills training course with the recording of emotional data and subjective ratings of communication skills.

\subsubsection{Method}

A total of 17 participants were recruited. All participants were over the age of 18 years old and were mid-late stage $\mathrm{PhD}$ students with 'none' to 'some' media interviewing experience.

Initially, participants engaged in a 1-hour introduction to media skills training and then a 2-hour training session. Participants' training sessions included two interviews; a radio interview followed by a face-toface interview and were provided feedback about their performance from the trainer.

Participants and trainers filled in a CSRS after each interview. Later, interviews were further rated by three neutral observers. Participants were asked if they would like to return to give their opinion on different methods of providing feedback.

\subsubsection{Data Investigation}

The outcome of this stage identified the emotional signals that are essential for a media interview. To meet the aim of the exploratory stage, this section describes how the data was analysed.

The researchers examined the internal consistency of the CSRS using Cronbach's Alpha (Makhoul, 1975). The degree of agreement of ratings by neutral observers was assessed using intraclass correlation (Bartko, 1966).

A weighted average was calculated using a composite score of the communication skills ratings where $40 \%$ was attributed to the trainer and $20 \%$ for each of the three neutral observers (Naim et al., 2015). This was done for the purpose of labelling the data into good and bad categories (cut-off defined by data mid-point). This established the ground-truth for the k-nearest neighbour (KNN) algorithm.

The relationships between patterns of emotional / non-verbal signals and participant performance evaluations were explored using KNN. The results of this will reveal the social signals that are important for a media interview.

A $t$-test was used to determine whether training gains are measurable between the radio interview and the face-to-face interview. The effect size obtained will give an indication of possible effect sizes for this type of intervention and will inform the sample size for the experiment stage (Erdfelder, Faul and Buchner, 1996). 


\subsection{Feedback Design Stage}

The aim of this stage was to establish the best method of providing feedback to participants about their performance in an understandable and actionable way. To assess the research aim, researchers employed a mixed-design approach.

Researchers called back volunteer participants from the exploratory stage to observe their interviews with data presented in various display formats. Their views on the most effective means to provide feedback were collected by means of a usability scale and semi-structured interviews.

\subsubsection{Method}

A sample of 5 was recruited as any usability concerns are identified with four to five participants, additional participants are less likely to reveal novel information (Virzi, 1992). Several methods of presenting feedback to participants were developed. After each method was presented participants rated each method of feedback using the SUS. After each channel of communication was complete participants engaged in a semi-structured interview. Interview prompts can be seen in Table 1 .

Table 1: Interview prompts for identifying views on each feedback method

\begin{tabular}{|l|l|}
\hline No. & Question \\
\hline 1. & $\begin{array}{l}\text { Please tell me which of the versions of the } \\
\text { design you liked the best? }\end{array}$ \\
\hline 2. & $\begin{array}{l}\text { Why did you like this version better than the } \\
\text { others you looked at? }\end{array}$ \\
\hline 3. & $\begin{array}{l}\text { Were there any elements of the less preferred } \\
\text { version that you liked? If so, what? }\end{array}$ \\
\hline 4. & $\begin{array}{l}\text { Were there any elements of your preferred } \\
\text { design that you don't like? If so, what? }\end{array}$ \\
\hline
\end{tabular}

\subsubsection{Feedback Display Methods}

There were several different methods for displaying feedback to participants about their performance for each channel of communication. Each are discussed.

\section{Facial expression}

There were three methods of presenting facial expression feedback. The first method was a simple playback of video with an emotion dashboard provided by iMotions displaying the 7 basic emotions (see example in Figure 1).

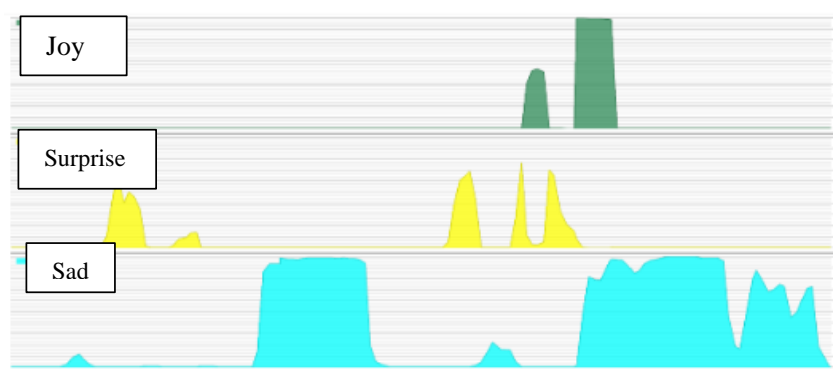

Figure 1: Facial expression feedback using iMotions emotional dashboard (Method 1)
The second method was a custom software was developed and was implemented using MATLAB (www.mathworks.com, release R2015a 8.5.0.197613, 64 bit) as a means of processing matrix-based data structures. The code corresponds to a basic implementation of an 'Emotion Dashboard' designed for customisable visualisation of key data from the emotion sensors (see Figure 2).
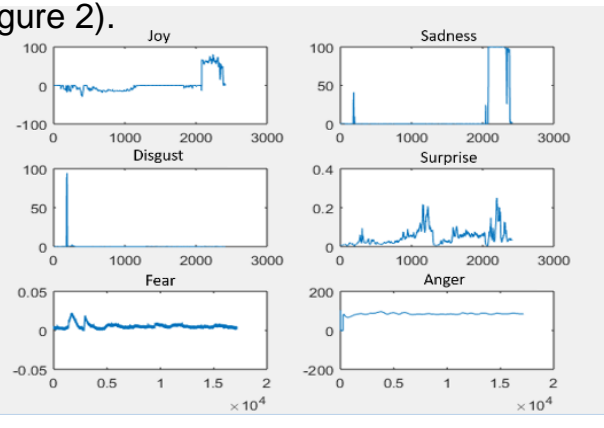

Figure 2: Facial expression feedback using developed emotional dashboard (Method 2)

The third and final method for facial expression feedback was a bar chart style template (see Figure 3). The participants actual performance was plotted alongside a 'good' performance value. All data obtained was normalised to the mean and standard deviation of each feature. Good performance was identified in the exploratory stage as part of the classification method.

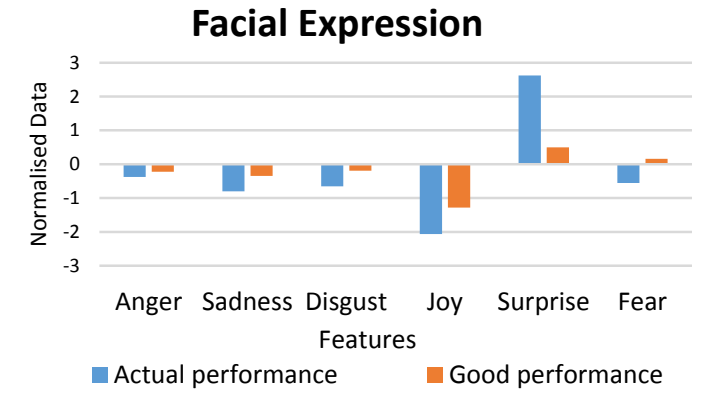

Figure 3: Facial expression feedback using a bar chart layout (Method 3)

\section{Layered Voice Analysis}

There were two methods of presenting feedback of voice emotion to participants. The first was the 'Emotion Diamond' method that is presented in real time on the Nemesysco ${ }^{\text {Ltd }}$ platform (see Figure 4). The second method can be observed in Figure 3.

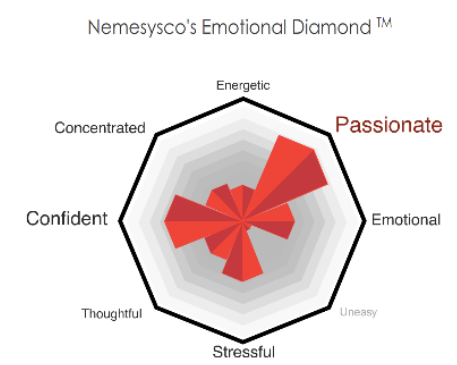

Figure 4: Voice emotion recognition feedback using the 'Diamond' shape (Method 1) 
Sociometric Badges, Gestures and Physiological Recordings

The only method for presenting feedback to participants for the sociometric badges was the bar chart style template. Participant feedback for gestures was by means of video playback with the bar chart style template presented at the end of playback.

The physiological recordings that were recorded in the exploratory stage were not fed back to participants as this is difficult for participants to action and therefore does not present as a method for feedback on performance.

\subsubsection{Method Identification and Interview Coding}

\section{System Usability Scale}

The SUS yields a single number representing a composite measure of the overall usability of the display method presented. To calculate this, items $1,3,5,7$ and 9 is the score contribution position, minus 1 . For items $2,4,6,8$ and 10 , the contribution is 5 minus the scale position. The sum of these scores were multiplied by 2.5. This was calculated for each feedback method (Brooke, 1996). These results were then summed and averaged for each method to gain an understanding of how participants rated each system overall.

\section{Interview Theme Identification}

Interviews were transcribed verbatim. Sematic themes were identified within the explicit or surface meanings of the data. Themes identified has not been interpreted beyond what has been transcribed and was identified in an inductive (bottom up) approach (Frith and Gleeson, 2004). Coding and theme analysis was completed according to Braun and Clarke's (2006) guidelines.

\subsection{Experimental stage}

The experiment was designed to compare performance of those who received media training with automated technology feedback to those that received traditional feedback.

The experimental hypotheses are:

1) There will be significant improvements in performance (as measured subjectively) from pre-test interview to post-test interview in both training conditions (main effect of training);

2) There will be significant changes in observed social signals detected between pre-test interview and post-test interview in both training conditions (main effect of training);

3) Training gains (as measured subjectively) from pre-test to post-test interview will be greater for the experimental condition (interaction effect);

4) Greater changes in social signals will be detected between pre-test interview and post-test interview for the experimental condition (interaction effect).

\subsubsection{Method}

According to the sample estimation calculation in the exploratory stage, a total of 22 participants were recruited. All participants were over the age of 18 years old and were mid-late stage PhD students with 'none' to 'some' media interviewing experience.

Initially, participants engaged in a baseline interview (face-to-face). Participants were paired according to gender, native language and baseline performance, they were then randomly assigned to a group by a flip of a coin. The automated / emotional feedback group received automated feedback and traditional feedback by the trainer and the traditional feedback group received traditional feedback by the trainer only.

A 30-minute pre-recorded introduction to media skills was then presented to participants. Subsequently, participants engaged a training session which encompassed a radio interview, face-face interview followed by a down-the-line interview. The difficulty of questions increased with each interview. Participants engaged in a post-test interview which mimicked question difficulty observed in the baseline interview. Additionally, participants filled in a self-evaluation of skills and confidence post-training containing two questions:

1) Please indicate on the scale below how much you estimate your skill in giving a media interview has improved over the course of today's training event.

2) Please indicate on the scale below the extent to which your confidence in giving a media interview has improved over the course of today's training event.

Questions were each rated on a five-point scale labelled as follows: 'no improvement', 'slight improvement', 'some improvement', 'considerable improvement' and 'great improvement'. This 2-item scale was designed for this study.

Emotional feedback was provided by the experimenter (with the trainer blind to whether participant received this feedback intervention or not). Participants were given feedback based on which areas needed the most improvement by observing the results presented in the bar chart. The number of feedback points varied depending on the individual performance of the participant. Participants only received feedback on signals where bar chart performance fell outside the 
minimum and maximum values defining 'good' performance on the basis of the exploratory study.

After each interview, participants were provided feedback from the trainer. Interviews were then played back to participants and then the bar chart summary was presented by the experimenter.

\section{Qualitative Interviews}

Lastly, semi-structured interviews were conducted. Probing questions for participants that received both traditional and emotional / social signal feedback and traditional feedback only can be seen in Table 2. Additional probing questions for participants that received both traditional and emotional / social signal feedback can be seen in Table 3.

Table 2: Questions for all participants

\begin{tabular}{|l|}
\hline 1. How did you feel about the feedback you received on \\
your performance today? \\
\hline 2. What, if anything, did you feel you were able to \\
change about how you presented yourself today \\
based on the feedback that you received?
\end{tabular}

Table 3: Questions for traditional and emotional / social signal group

1. How did you feel about seeing the system feedback of your use of emotional signals and body language during the course of the training session today?

2. Do you feel that the system feedback helped you appreciate aspects of your performance that you might not have noticed by just watching / hearing the playback?

3. To what extent did you feel that the feedback you received was 'actionable' - that is to what extent did you think you could control or improve the behaviour which was highlighted in feedback?

4. Could the feedback have been presented in a clearer way? If so, what would you change about the way the feedback was presented?

5. Were there additional aspects of your non-verbal behaviour that you think it would have been useful to receive feedback on? If so, which?

6. Do you have any further comments about your experiences or the way that the feedback was presented today?

\subsubsection{Data Analysis}

\section{Subjective Ratings of Communication Scores}

The experimental hypotheses that relates to subjective judgements of participant performance were tested by means of $2 \times 2$ mixed factorial ANOVAs. The data had two independent variables, each with two levels: Interview session (within participants: baseline and post-test interviews) and feedback type (between-subjects: emotion / social signal and traditional feedback condition). The dependent variable was communication skills ratings.

Between-participants $t$-tests were run to explore whether there were any significant differences in participant post-training self-evaluation of skill and confidence. This was done across the two feedback conditions (using the self-rating of training gain scores).

\section{Social Signals}

Social signal experimental hypotheses were tested by means of a factor analysis to identify the combinations of social signals necessary for a media interview. Subsequently, mixed-model ANOVA was applied to the selected factors with a view to identify cases in which there was a differential pre- post difference with respect to the experimental group.

The social signal data had two independent variables: feedback type (between-participants variable with two levels: Empatic feedback or nonEmpatic feedback) and timing of interview (withinparticipants variable with two levels: pre-test and post-test interview). The dependent variables were all the social signal recordings produced by the software that were recorded during the interview.

\section{Qualitative Interviews: Theme Identification}

Interviews were transcribed and analysed as in the exploratory stage.

\section{RESEARCH CONTRIBUTIONS}

The short-term contributions of the current research will; provide evidence of meaningful measures of training performance, increase knowledge on how to feedback data on someone's use of signals that is easily understood and actionable and increase knowledge of the potential training benefit associated with provision of such feedback in context of media skills training. In addition, the long-term benefit will lead to the development of a training avatar.

\section{Acknowledgements}

The author acknowledges support of the UK Defence Science and Technology Laboratory (Dstl).

\section{REFERENCES}

Bartko, J. J. (1966) 'The Intraclass Correlation Coefficient as a Measure of Reliability', Psychological Reports. SAGE Publications Sage CA: Los Angeles, CA, 19(1), pp. 3-11. 
Bennett, K. B. and Flach, J. M. (1992) 'Graphical Displays: Implications for Divided Attention, Focused Attention, and Problem Solving', Human Factors: The Journal of the Human Factors and Ergonomics Society, 34(5), pp. 513-533.

Brooke, J. (1996) 'SUS - A quick and dirty usability scale', Usability Evaluation in Industry, 189(194), pp. 4-7.

Bush, L. K. et al. (1989) 'The effects of facial control and facial mimicry on subjective reactions to comedy routines', Motivation and Emotion, 13(1), pp. 31-52.

Cerrato, L. (2005) 'Linguistic functions of head nods', in Proc. Conf. Multi--modal Communication, pp. 137-152.

Condon, W. S. and Ogston, W. D. (1966) 'Sound film analysis of normal and pathological behavior patterns', Journal of Nervous and Mental Disease, 143(4), pp. 338-347.

Ekman, P. (1992) 'Facial expressions of emotion: New findings, new questions', Psychological science. SAGE Publications Sage CA: Los Angeles, CA, 3(1), pp. 34-38.

Ekman, P. and Friesen, W. (1982) 'False, felt, and miserable smiles', Journal of Nonverbal Behavior, 6(4), pp. 238-258.

Ekman, P. and Friesen, W. V. (1972) 'Hand Movements', Journal of Communication. Oxford University Press, 22(4), pp. 353-374.

Ekman, P. and Friesen, W. V. (1978) The Facial Action Coding System: a technique for the measurement of facial movement. Consulting Psychologists, San Francisco.

Ellis, D. G., Duran, R. L. and Kelly, L. (1994) 'Discourse Strategies of Competent Communicators: Selected Cohesive and Linguistic Devices', Research on Language and Social Interaction, 27(2), pp. 145-170.

Erdfelder, E., Faul, F. and Buchner, A. (1996) 'GPOWER: A general power analysis program', Behavior Research Methods, Instruments, \& Computers, 28(1), pp. 1-11.

Fiske, J. (2010) Introduction to Communication Studies. Routledge.

Hall, E. (1969) 'The Hidden Dimension: man's use of space in public and in private', Anchor Books. New York, p. 217.
Hurley, C. M. and Frank, M. G. (2011) 'Executing Facial Control During Deception Situations', Journal of Nonverbal Behavior, 35(2), pp. 119131.

Kendon, A. (2004) Gesture: Visible action as utterance. Cambridge: Cambridge University Press.

Kortum, P. T. and Bangor, A. (2013) 'Usability Ratings for Everyday Products Measured with the System Usability Scale', International Journal of Human-Computer Interaction. Taylor \& Francis Group, 29(2), pp. 67-76.

Likas, A., Vlassis, N. and J. Verbeek, J. (2003) 'The global k-means clustering algorithm', Pattern Recognition. Pergamon, 36(2), pp. 451-461.

Makhoul, J. (1975) 'Linear Prediction: A Tutorial Review', Proceedings of the IEEE. Midwest Research-to-Practice Conference in Adult, Continuing, and Community Education, 63(4), pp. 561-580.

Marzillier, J. S. (1976) 'Gaze and mutual gaze', Behaviour Research and Therapy, 14(6), p. 486.

McNeill, D. (1992) Hand and Mind. Chicago: Chicago Press.

Naim, I. et al. (2015) 'Automated Analysis and Prediction of Job Interview Performance', IEEE Transactions on Affective Computing, pp. 1-14.

Oatley, K. and Johnson-Laird, P. N. (1987) 'Towards a Cognitive Theory of Emotions', Cognition and Emotion, 1(1), pp. 29-50.

Pentland, A. and Heibeck, T. (2008) Honest Signals: How They Shape Our World. Cambridge, Massachusetts: MIT Press.

Poggi, I. and D 'errico, F. (2011) 'Social signals: A psychological perspective', in Computer Analysis of Human Behaviour. London: Springer, pp. 185-225.

Rasmussen, J. and Vicente, K. K. J. (1989) 'Coping with Human Errors through System-Design Implications for Ecological Interface Design', International Journal of Man-Machine Studies, 31(5), pp. 517-534.

Spitzberg, B. H. and Adams, T. W. (2007) 'CSRS, the Conversational Skills Rating Scale: An Instructional Assessment of Interpersonal Competence', NCA, National Communication Association. 
Taylor, R. (2015) Media Interview Techniques: A complete guide ot media training. London: Kogan Page.

Tracy, J. L. et al. (2015) 'The nonverbal communication of emotions', Current Opinion in Behavioral Sciences, 3, pp. 25-30.

Vicente, K. J. and Rasmussen, J. (2014) 'The Ecology of Human-Machine Systems 11: Mediating \&quot;Direct Perception\&quot; in Complex Work Domains', Ecological Psychology, 2(3), pp. 207-249.

Vinciarelli, A., Pantic, M. and Bourlard, H. (2009) 'Social signal processing: Survey of an emergin domain', Image and Vision Computing, 27(November 2008), pp. 1743-1759.

Virzi, R. A. (1992) 'Refining the Test Phase of Usability Evaluation: How Many Subjects Is Enough?', Human Factors: The Journal of the Human Factors and Ergonomics Society. SAGE PublicationsSage CA: Los Angeles, CA, 34(4), pp. 457-468.

Vrij, A., Edward, K. and Roberts, K. P. (2000) 'Detecting deceit via analysis of verbal and nonverbal behavior', Ray Bull Journal of Nonverbal Behavior Winter, 24(4).

Woods, D. D. (1991) 'The Cognitive Engineering of Problem Representations', in Weir, G. R. S. and Alty, J. L. (eds) Human-Computer Interaction and Complex Systems. London: Academic, pp. 169-188. 